\title{
artigo
}

Freitas, K.A.B.S.; Lima, T.O. Castro, A.B.; Batista da Silva, K.A.; Vigliassi, A.P.; Popim, R.C.;

Elaboração e implantação de protocolo de condutas no extravasamento de medicamentos não quimioterápicos

\section{Elaboração e implantação de protocolo de condutas no extravasamento de medicamentos não quimioterápicos}

\author{
Elaboration and implementation of protocol of conducts in the extraction of non-chemotherapic medicines \\ Elaboración e implementación de protocolo de conductas en la extracción de medicamentos no quimioterápicos
}

\begin{abstract}
RESUMO
Objetivo: Descrever a experiência na elaboração e implantação de um protocolo de condutas frente ao extravasamento de medicamentos não quimioterápicos. Método: Pesquisa descritiva, do tipo relato de experiência, realizada após a implantação do Time de Cateter de um Hospital Público, de nível quaternário, do interior do Estado de São Paulo, com a finalidade de elaboração de protocolo assistencial frente ao extravasamento de medicamentos não quimioterápicos. As ações foram divididas em quatro fases, que vão desde o conhecimento do time, até a mensuração dos resultados. Realizada pesquisa na literatura recente para construção do protocolo. Resultados: 0 protocolo foi elaborado, revisado e validado. Traz os principais sinais e sintomas que podem ocorrer, um quadro com as medicações vesicantes e qual a compressa utilizar, além de um fluxograma que norteia a ação. Conclusão: Novos conhecimentos baseados em evidência foram adquiridos e a habilidade foi melhorada, proporcionando mais segurança durante um atendimento de extravasamento.
\end{abstract}

DESCRITORES: Extravasamento de Materiais Terapêuticos e Diagnósticos; Cuidados de Enfermagem; Protocolos de Enfermagem; Relato de Experiência.

\section{ABSTRACT}

Objective: To describe the experience in the elaboration and implementation of a protocol of conducts against the overflow of non-chemotherapeutic drugs. Method: Descriptive research, of the type of experience report, carried out after the implantation of the Catheter Team of a Public Hospital, of a quaternary level, in the interior of the State of São Paulo, with the purpose of elaborating an assistance protocol in view of the overflow of non-medications chemotherapy. The actions were divided into four phases, ranging from the knowledge of the team, to the measurement of results. Research in recent literature was carried out to build the protocol. Results: The protocol was developed, reviewed and validated. It brings the main signs and symptoms that may occur, a table with vesicant medications and which compress to use, in addition to a flow chart that guides the action. Conclusion: New evidence-based knowledge was acquired and the skill was improved, providing more security during an overflow care.

DESCRIPTORS: Extravasation of Therapeutic and Diagnostic Materials; Nursing care; Nursing protocols; Experience report.

\section{RESUMEN}

Objetivo: Describir la experiencia en la elaboración e implementación de un protocolo de conductas contra el desbordamiento de fármacos no quimioterápicos. Método: Investigación descriptiva, del tipo de relato de experiencia, realizada luego de la implantación del Equipo de Catéter de un Hospital Público, de nivel cuaternario, en el interior del Estado de São Paulo, con el propósito de elaborar un protocolo de atención ante la extravasación de no medicamentos quimioterapia. Las acciones se dividieron en cuatro fases, que van desde el conocimiento del equipo, hasta la medición de resultados. Se llevó a cabo una investigación en la literatura reciente para construir el protocolo. Resultados: El protocolo fue desarrollado, revisado y validado. Trae los principales signos y síntomas que pueden presentarse, una tabla con medicamentos vesicantes y los que se comprimen a utilizar, además de un diagrama de flujo que guía la acción. Conclusión: Se adquirieron nuevos conocimientos basados en la evidencia y se mejoró la habilidad, brindando más seguridad durante una atención de desbordamiento.

DESCRIPTORES: Extravasación de Materiales Terapéuticos y de Diagnóstico; Cuidado de Enfermera; Protocolos de Enfermería; Informe de Experiencia.

RECEBIDO EM: 10/09/2020 APROVADO EM: 30/09/2020 


\section{Karina Alexandra Batista da Silva Freitas}

Enfermeira mestre e doutoranda pelo Departamento de Enfermagem da Faculdade de Medicina de Botucatu- UNESP. Supervisora do Ambulatório de Oncologia do Hospital Estadual Botucatu. Botucatu (SP).

ORCID ID: 0000-0002-3413-8468

\section{Talita Oliveira de Lima}

Mestre pelo Departamento de Enfermagem da Faculdade de Medicina de Botucatu- UNESP. Enfermeira assistencial do Ambulatório de Oncologia do Hospital Estadual Botucatu. Botucatu (SP).

ORCID ID: 0000-0002-6617-6514

\section{Andrezza Beluomini de Castro}

Mestre e doutoranda pelo Departamento de Enfermagem da Faculdade de Medicina de Botucatu- UNESP. Gerente de Enfermagem do Hospital Estadual Botucatu. Botucatu (SP).

ORCID ID: 0000-0002-5161-4315

\section{Karen Aline Batista da Silva}

Doutora pelo Departamento de Enfermagem da Faculdade de Medicina de Botucatu- UNESP. Diretora do Núcleo de Apoio à Superintendência Hospital das Clínicas da Faculdade de Medicina de Botucatu (SP).

ORCID ID: 0000-0002-8643-5333

\section{Alessandra Passarelli Vigliassi}

Enfermeira especialista em oncologia, membro do TIME de cateter do Hospital das Clínicas da Faculdade de Medicina de Botucatu- UNESP. Enfermeira assistencial do Ambulatório de Oncologia do Hospital Estadual Botucatu. Botucatu (SP).

ORCID ID: 0000-0002-4651-888X

\section{Regina Célia Popim}

Professora associada do Departamento de Enfermagem da Faculdade de Medicina de Botucatu- UNESP.

ORCID ID: 0000-0001-8341-1590

\section{INTRODUÇÃO}

0 tema segurança do paciente tomou visibilidade após a publicação do To Err is Human, pelo Institute of Medicine, em 1999, que abordou o impacto dos eventos adversos (EA) nas instituições e na vida dos pacientes. Definiu que o EA é todo dano causado pelo cuidado à saúde, não associado à patologia responsável pela internação/atendimento ${ }^{1}$.

A via parenteral de administração de medicamentos é comumente utilizada em situações que requerem uma resposta sistêmica rápida. No entanto, existem riscos crescentes e iatrogenias durante o seu uso, como por exemplo o extravasamento ${ }^{2}$.

Os medicamentos são classificados em irritantes e vesicantes. A definição de infiltração e extravasamento está diretamente relacionada ao tipo de medicação utilizada. O extravasamento é definido como a infusão de medicamentos vesicantes para fora do vaso sanguíneo, acometendo tecidos circunvizinhos, podendo danificar o tecido mole, nervos, tendões, causando bolhas e necrose e uma intensa reação inflamatória, já a infiltração está relacionada à infusão de medicação irritante, podendo causar uma reação inflamatória local, não sendo diretamente tóxica aos tecidos ${ }^{3,4}$. Neste artigo, abordaremos somente os extravasamentos.

Os principais sinais e sintomas são: vermelhidão, edema, ausência de retorno venoso, parada na infusão, ardor, queimação e a dor pode ou não estar presente. Porém é possível que o paciente não apresente sintomas ou que esses sejam muito leves 3 . A incidência pode chegar a $39 \%$ em pacientes adultos e $70 \%$ em crianças. Em até $25 \%$ dos casos a morbidade decorrente do extravasamento é mais grave do que a causa base da internaçãó .

O mecanismo do dano tecidual depende de vários fatores como: osmolaridade, $\mathrm{pH}$ e mecanismo de ação do medicamento2. Medicamentos com pH fora da faixa de 5,5 a 8,5 e aqueles com osmolaridade superior a 600 são os mais propensos a causar danos. $\mathrm{O}$ diluente do medicamento também pode ser a causa do dano vascular, como os ingre- dientes inativos álcool benzílico e propileno glicol podem induzir respostas inflamatórias e causar a lesão $o^{2.5}$. Importante afirmar que independente da medicação extravasada pode ocorrer a síndrome compartimental com possibilidade de dano arterial e venoso podendo levar a amputação da extremidade ${ }^{6,7}$.

Os fatores de risco estão relacionados com a escolha do dispositivo, tipo de medicamento administrado, localização do cateter, duração da infusão, doenças vasculares periféricas, extremos de idade, estado mental alterado, linfedema, técnica incorreta de punção venosa, administração de medicamentos vesicantes por acesso venoso periférico ${ }^{8}$. Os locais mais frequentemente envolvidos no extravasamento são o dorso da mão e do pé, fossa antecubital e áreas com pouco tecido subcutâneo ${ }^{6}$.

Não existe tratamento padrão para a fase aguda da lesão. Porém, uma vez detectado, alguns cuidados devem ser realizados imediatamente. A infusão deve ser interrompida e deve-se aspirar o máximo de medicamento possível por meio do dispositivo de punção. Após a remoção da agulha, o membro deve ser 


\section{artigo}

Quadro 1. Descrição dos medicamentos vesicantes e indicação de compressas² MEDICAMENTO

Aminofilina $24 \mathrm{mg} / \mathrm{ml}$

Amiodarona 50mg/ml

Bicarbonato de sódio 8,4\%

Cafeina, citrat $20 \mathrm{mg} / \mathrm{ml}$

Cloreto de Potássio 19,1\%

Cloridrato de esmolol $10 \mathrm{mg} / \mathrm{ml}$.

Contraste $\mathrm{p} /$ ressonância magnet gadolino

Contraste não ionico p/tomo/hemod c/teor de 350mgi

Contraste $\mathrm{p} /$ resson gadoverset 330,9mg/

Diazepam $5 \mathrm{mg} / \mathrm{ml}$

Dobutamina $12,5 \mathrm{mg} / \mathrm{ml}$

Dopamina $5 \mathrm{mg} / \mathrm{ml}$

Epinefrina $1 \mathrm{mg} / \mathrm{ml}$

Fenilefrina $10 \mathrm{mg} / \mathrm{ml}$

Fenitoína $50 \mathrm{mg} / \mathrm{ml}$

Fenobarbital 100mg/ml

Gliconato de cálcio 10\%

Glicose 10\%

Glicose $50 \%$

Glicose $75 \%$

Haloperidol 5mg/ml

Haloperidol decanoato $50 \mathrm{mg} / \mathrm{ml}$

Hemitartarato de Norepinerina, $2 \mathrm{mg} / \mathrm{ml}$

Manitol 20\%

Polimixina b

Prometazina $25 \mathrm{mg} / \mathrm{ml}$

Propofol 10mg/ml

Propofol $1 \%$

Propofol $2 \%$

Sulfato de efedrina $50 \mathrm{mg} / \mathrm{ml}$

Vancomicina 500mg

Vasopressina 20U/ml

Alprostadil

Cloreto de cálcio

Nutrição Parenteral

Fonte: Protocolo de Extravasamento de Medicamentos não Quimioterápicos. Hospital das Clínicas da Faculdade de Medicina de Botucatu - UNESP. 2020.

elevado a fim de minimizar o edema e favorecer a drenagem linfática ${ }^{8}$.

A aplicação de compressas deverá ser escolhida de acordo com o fármaco extravasa-
COMPRESSA

Fria

Quente

Quente

Fria

Quente

Fria

Fria

Fria

Fria

Fria

Quente

Quente

Quente

Fria

Quente

Fria

Fria

Fria

Fria

Fria

Fria

Fria

Quente

Fria

Fria

Fria

Fria

Fria

Fria

Quente

Fria

Quente

Fria

Fria

Fria 
Figure 1. Flowchart for identification, selection and inclusion of integrative review studies. Niterói, RJ, Brazil, 2020.

Suspeita de extravasamento
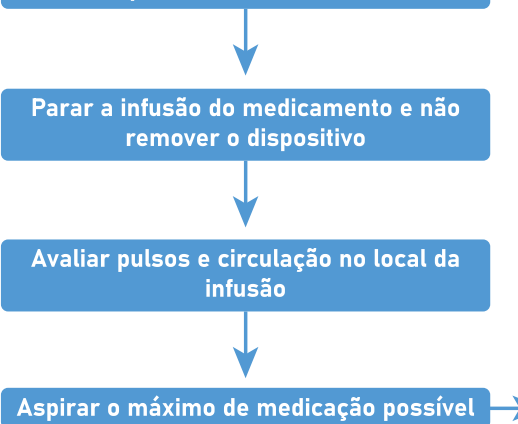

Retirar o dispositivo de punção
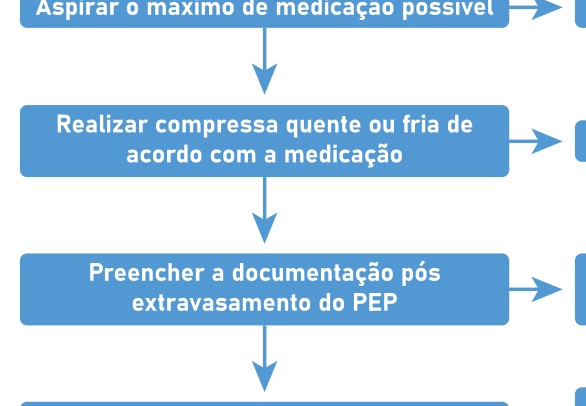

Realiza notificação de enventos adversos
Administrar hialuronidase tópica $3 \mathrm{x}$ dia

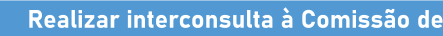
Curativos

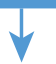

Acompanha o paciente na internação e ambulatoialmente

Fonte:Protocolo de Extravasamento de Medicamentos não Quimioterápicos. Hospital das Clínicas da Faculdade de Medicina de Botucatu UNESP. 2020.

no extravasamento de medicamentos não quimioterápicos, surgiu a necessidade de elaboração de um protocolo baseado em evidências que garantisse o acompanhamento desse paciente até a resolução completa da lesão.

Assim, delineou-se a seguinte questão da pesquisa: qual a melhor conduta a ser tomada, imediatamente, frente a um extravasamento de medicamento não quimioterápico?

O extravasamento de medicamentos antineoplásico não foi abordado, pois a instituição, palco do estudo, já possuía protocolo instituído com condutas específicas, baseadas em vasta literatura, e método que não se aplicam a medicamentos não quimioterápicos.

\section{MÉTODO}

Pesquisa descritiva, do tipo relato de experiência, realizada após a implantação do Time de Cateteres de um Hospital Público, de nível quaternário, do interior do Estado de São Paulo, com a finalidade de elaboração de protocolo assistencial frente ao extravasamento de medicamentos não quimioterápicos.
O protocolo foi construído de acordo com modelo padrão da instituição, fornecido pelo escritório de qualidade.

As ações foram divididas em 4 fases. $\mathrm{Na}$ fase 1 , foi realizado o levantamento do conhecimento do Time de Cateteres frente ao extravasamento de medicamentos não quimioterápicos. $\mathrm{Na}$ fase 2 , foi realizada busca na literatura nas bases de dados PubMed e Google Scholar de estudos que norteassem as condutas tomadas frente ao extravasamento e elaboração do protocolo. $\mathrm{Na}$ fase 3 , será realizada a instrumentalização da equipe de enfermagem para detecção e conduta frente ao protocolo elaborado. A fase 4, contemplará o levantamento e avaliação dos indicadores realizados para mensurar as condutas frente ao extravasamento, disponível no Prontuário Eletrônico do Paciente (PEP).

Cabe ressaltar que a Instituição já possui um Manual de Extravasamento de Antineoplásicos, com fluxo instituído de condutas, portanto foram excluídos os estudos que tratavam dessa temática.

Após a confecção, o mesmo foi revisado por outros dois membros do time que não participaram da elaboração. Posteriormente, após realizada as modificações sugeridas, foi validado pelo escritório de qualidade e divulgado a todos os colaboradores por meio do sistema eletrônico onde estão agrupados todos os protocolos institucionais.

Foi estabelecido que cabe ao enfermeiro todo o gerenciamento do extravasamento, bem como a prescrição do antídoto e das condutas conforme o protocolo pré-estabelecido.

\section{RELATO DE EXPERIÊNCIA}

O protocolo foi escrito de uma forma clara, concisa e de fácil entendimento trazendo os principais sinais e sintomas, além de um quadro (1) que identifica as medicações vesicantes e qual a compressa utilizar.

Para facilitar o atendimento foi realizado um fluxograma que traz as principais condutas a serem utilizadas, como demonstra a Figura 1.

O protocolo traz que deverão ser adotadas medidas preventivas como: uso do fluxograma de passagem de acesso central no momento da internação do paciente, avaliação da rede venosa para a melhor escolha de veias calibrosas, realizar checagem do acesso venoso quanto ao fluxo/refluxo antes de administrar o medicamento, evitar punção venosa em fossa antecubital, veia jugular, membros que correspondam ao lado da mastectomia, edemaciados, sem sensibilidade, membros inferiores. Proíbe a utilização de cateter agulhado para punção venosa.

O extravasamento também poderá ocorrer em cateter totalmente implantado, portanto, após avaliação da área, deverá ser realizada a escolha da agulha para punção. Os pacientes também fazem parte do processo de prevenção e deverão ser encorajados a observar os principais sinais e sintomas como queimação e dor.

Após o extravasamento, o enfermeiro deverá preencher a documentação, colocando informações como: data e hora da ocorrência, local de administração e medicamento extravasado, quantidade aspirada (ml), dispositivo utilizado e calibre, data da punção, técnica de administração: bolus, gravitacional ou bomba de infusão; intervenções realizadas, planejamento dos cuidados, sinais e sintomas apre- 


\section{artigo}

Freitas, K.A.B.S.; Lima, T.O. Castro, A.B.; Batista da Silva, K.A.; Vigliassi, A.P.; Popim, R.C.;

Elaboração e implantação de protocolo de condutas no extravasamento de medicamentos não quimioterápicos

sentados e realizar a Notificação de Eventos Adversos, ambos disponíveis no PEP.

\section{DISCUSSÃO}

A assistência de enfermagem deve ser baseada em evidências e padronizações adequadas para que não haja ações de negligência, imperícia e imprudência, podendo causar danos aos pacientes, problemas legais e éticos aos profissionais além de descréditos da classe pela sociedade?.

A segurança do paciente está relacionada à assistência de enfermagem e o uso de protocolos adequados devem ser encorajados e também mensurados.

Para a elaboração de um protocolo deve-se, inicialmente, realizar um diagnóstico situacional, fazendo um mapeamento do seu território e verificando as reais necessidades daquela área, contemplando o perfil da enfermagem, do usuário e os riscos inerentes ao seu tratamento ${ }^{12}$. Em nosso estudo, foi observado a deficiência de conhecimento da equipe de enfermagem quanto ao extravasamento de medicamentos não quimioterápicos.

O Conselho Federal de Enfermagem instituiu Resoluções, que amparam a atuação dos profissionais e recomenda-se que as atribuições dos profissionais de enfermagem sejam descritas em todos os protocolos assistenciais instituídos, visando à garantia do seguimento das legislações vigentes e à organização do processo de trabalho da equipe de enfermagem ${ }^{13}$.

A documentação deve ser apropriada, pois demonstra a prática dos cuidados de enfermagem, devendo o registro deve ser realizado constando todas as etapas do cuidado. $\mathrm{O}$ seguimento do paciente também deverá ser realizado. Além disso, pode auxiliar no diagnóstico da necessidade de treinamentos específicos ${ }^{14}$. Na nossa Instituição o seguimento do paciente é realizado pelos membros da Comissão de Curativos, formada por enfermeiros capacitados no tratamento de feridas e que podem dar as melhores condutas nesses casos.

Acreditamos que com a elaboração do protocolo, estabeleceremos uma nova estratégia no atendimento do extravasamento, havendo uma mudança na nossa conduta e de toda equipe de enfermagem, frente a esse evento. Aprendemos novos conhecimentos baseados em evidência, bem como melhoramos nossa habilidade

\section{CONCLUSÃO}

A elaboração do protocolo de extravasamento de medicamentos não quimioterápicos pode proporcionar uma assistência segura, evitando danos irreparáveis que podem interferir na qualidade de vida do paciente, além de instrumentalizar e direcionar a avaliação, prescrição do antídoto e condutas, ao profissional enfermeiro.

\section{REFERÊNCIAS}

1. Kohn LT, Corrigan JM, Donaldson MC, editors. Committee on Quality of Health Care: Institute of Medicine. To Err is Human: building a safer health system. Washington (DC): National Academy Press:2000.

2. Le A. Extravasation of Noncytotoxic Drugs: A Review os the Literature. Annals of Phamacotherapy, 2014, 48(7) 870-886. DOI: $10.1177 / 1060028014527820$

3. Hannon MG, Lee SK. Extravasation Injuries. The Journal of Hand Surgery. 2011 36(12) 2060-2065.

4. Al-Benna S, O'Boyle C, Holley J. Extravasation Injuries in Adults. ISRN Dermatol. 2013. DOI: 10.1155/2013/856541

5. Czerwonka E. Guideline for Non - Chemotherapeutic Agents: Prevention and Treatment of Chemical Phlebits and Extravasation of Peripherally Administered Non - chemotherapeutic Agents Adult/Pediatric - Inpatient Clinical Practice Guideline. University of Wisconsin Hospitals. 2015.

6. Milchesi DA, Mota WM, Lobato RC, Monteiro Jr AA, Gemperli R. Opções de tratamento cirúrgico em lesões cutâneas por extravasamento acidental de drogas: experiência do Hospital das Clínicas da Faculdade de Medicina da USP. Rev Col Bras Cir. 2018;45(4):e1912. DOI: 10.1590/0100-6991e-20181912.

7. Gorski L, Hadawai L, Hagle ME, McGoldrick M, Orr M, Doellman D. Infusion Therapy. Standards of Practice. Journal of Infusion Nursing. Supplement to January/February; 39(1S).2016.

8. Reynolds PM, MacLaren R, Mueller AW, Fish DN, Kiser TH. Management of Extravasation Injuries: A Focused Evaluation of Noncy- totoxic Medications. Pharmacotherapy. 2014; 34(6): 617-632.

9. Pimenta, Cibele A. de M...[et al.]. Guia para construção de protocolos assistenciais de enfermagem/ Cibele A. de M. Pimenta [et al.].; COREN-SP - São Paulo: COREN-SP, 2015. ISBN: 978-85-6872002-8.

10. Werneck MAF, Faria HP, Campos KFC. Protocolos de cuidado à saúde e de organização do serviço [Internet]. Belo Horizonte: Universidade Federal de Minas Gerais/Núcleo de Educação em Saúde Coletiva; 2009 [citado 2020 set. 28]. Disponivel em: https://www. nescon. medicina.ufmg.br/biblioteca/imagem/1750.pdf 2.

11. Manchikanti L, Singh V, Helm S 2nd, Trescot AM, Hirsch JA. A critical appraisal of 2007 American College of Occupational and Environmental Medicine (ACOEM) Practice Guidelines for Interventional Pain Management: an independent review utilizing AGREE, AMA, IOM, and other criteria. Pain Physician. 2008;11(3):291-310.

12. Silva CSSL, Koopmans FF, Daher DV. O Diagnóstico Situacional como ferramenta para o planejamento de ações na Atenção Primária a Saúde. Revista Pró- UniverSUS. 2016 Jan./Jun.; 07 (2): 30-33.

13. Brasil, Conselho Federal de Enfermagem. Diretrizes para elaboração de protocolos de Enfermagem na Atenção Primária à Saúde pelos Conselhos Regionais de Enfermagem/Conselho Federal de Enfermagem Brasilia: COFEN 2018.

14. Gozzo TO, Almeida TD, Cruz LAP. Notificação de Extravasamento de Agentes Quimioterápicos em um Hospital Universitário. Cienc Cuid Saude. 2018; 17(2). 